\title{
Phase-conjugated Pilots for Fibre Nonlinearity Compensation in CO-OFDM Transmission
}

\author{
Son Thai Le, Mary E. McCarthy, Naoise Mac Suibhne, Andrew D. Ellis and Sergei K. Turitsyn \\ (Invited paper)
}

\begin{abstract}
In this paper we demonstrate a novel fiber nonlinearity compensation technique for coherent optical orthogonal frequency division multiplexing (CO-OFDM) systems based on the transmission of phase-conjugated pilots (PCPs). In this scheme, a portion of OFDM subcarriers (up to $50 \%$ ) is transmitted with its phase-conjugates, which is used at the receiver to estimate the nonlinear distortions in the respective subcarriers and other subcarriers, which are not accompanied by PCPs. Simulation and experimental results show that, by varying the PCP overhead a performance improvement up to $4 \mathrm{~dB}$ can be achieved. In addition, the proposed technique can be effectively applied in both single polarization and polarization division multiplexed (PMD) systems, in both single channel and wave length division multiplexing (WDM) systems, thus, offering highest flexibility in implementations.
\end{abstract}

Index Terms -Nonlinearity mitigation, coherent detection, coherent optical transmission, orthogonal frequency division multiplexing.

\section{INTRODUCTION}

$\mathrm{T}$ heoretically the capacity of a fixed bandwidth Gaussian communication channel is logarithmically proportional to the signal-to-noise ratio [1]. As a result, the capacity of optical fiber communications channel should increase monotonically with the transmit signal power. However, the nonlinear distortion due to Kerr effect limits the maximum optical power that could be launched into an optical fiber (without degrading the system performance) [2, 3]. Fiber Kerr nonlinearity effect thus sets an upper bound on the achievable data rate in optical fiber communications using linear transmission techniques[4].

There have been extensive efforts in attempting to surpass the Kerr nonlinearity limit through several nonlinearity compensation techniques. Digital-back-propagation (DBP) is an effective nonlinearity compensation method, which removes the nonlinear distortion by inverting the distorted signal at the receiver digitally [5]. However, accurate DBP requires a substantial increase in digital signal processing (DSP) complexity, proportional to the number of spans.

Manuscript received October 06, 2014. S. T. Le, M. E. McCarthy, N. Mac Suibhne, A. D. Ellis and S. K. Turitsyn are with Aston Institute of Photonic Technologies (AIPT), School of Engineering and Applied Science, Aston Triangle, Birmingham, B4 7ET, UK (corresponding author phone: +44(0)744-702-40-09; e-mail: let1@aston.ac.uk). Copyright (c) 2014 IEEE. Personal use of this material is permitted. However, permission to use this material for any other purposes must be obtained from the IEEE by sending a request to pubs-permissions@ieee.org.
Furthermore, in wavelength-division multiplexed (WDM) systems the effectiveness of DBP is significantly reduced as the neighbouring WDM channels are unknown to the compensator.

Digital [6] and optical [7-9] phase conjugations (OPCs) at the mid link or installed at the transmitter [10] are other wellknown nonlinear compensation techniques that conjugate the signal phase after transmission in one segment of the link in order to achieve a net cancellation of the nonlinear phase shift using the nonlinearity generated in the second segment of the link. However, OPC modifies the transmission link by inserting a phase conjugator at the middle point of the link, and imposes significant symmetry conditions with respect to the phase conjugator, and thus, significantly reducing the flexibility in an optically routed network.

Recently, novel nonlinear compensation technique called phase-conjugated twin waves (PC-TW) has been proposed in [11]. PC-TW is a transponder-based technique that can be implemented with minimal additional optical hardware or DSP. In this scheme, the signal complex waveform and its phase-conjugate copy are simultaneously transmitted in $\mathrm{x}$ - and $y$-polarization states. If the dispersion map of the link is symmetrical, the nonlinear distortions on $\mathrm{x}$-and $\mathrm{y}$-polarizations are essentially anticorrelated, and thus, it can be subsequently mitigated at the receiver through coherent superposition of the two copies. The PC-TW provides a simple and effective solution in compensating optical fiber nonlinearity as it requires only an additional per symbol conjugate-and-add operation prior to symbol detection. However, the one serious shortcoming of PC-TW is that it sacrifices half the transmission capacity. To address this drawback of PCTW, a dual PCTW scheme has been also proposed for single carrier systems, yielding an improvement of $\sim 1.2 \mathrm{~dB}$ [12]. Unfortunately, quadrature pulse shaping is required for dual PCTW, which cannot be applied effectively for multicarrier modulation formats such as orthogonal frequency division multiplexing (OFDM). To address this problem, a phaseconjugated coding scheme has been proposed in [13], offering around $1.5 \mathrm{~dB}$ performance gain in BPSK CO-OFDM transmission.

The general idea of communication through phaseconjugated optical variants is described in [14], claiming that a meaningful performance gain can be achieved through coherent superposition of the signal and its phase conjugated copy, which can be transmitted in a different polarization state, time slot, spatial localization, optical carrier wavelength and 
subcarrier frequency. In [15], coherent superposition of the useful signal and its phase conjugated copy transmitted in a different optical wavelength was adopted to increase significantly the transmission distance. In addition, in [16] it was also applied to OFDM subcarrier pairs with Hermitian symmetry. However, in a similar manner to the PCTW scheme, both approaches proposed in $[15,16]$ also sacrifice half the transmission capacity.

In this paper we demonstrate a novel nonlinearity compensation technique for CO-OFDM systems based on the transmission of phase-conjugated pilots (PCPs), without scarifying $50 \%$ of the transmission capacity. The general idea was first presented in [17]. In this scheme, a portion of the OFDM subcarriers (up to 50\%) are transmitted as phaseconjugates of other subcarriers. The PCPs are used at the receiver to estimate the nonlinear distortion of their respective original subcarriers. The estimated distortion can also be used to compensate the nonlinear impairments in other subcarriers close to the PCP, thanks to the narrow OFDM subcarrier spacing (tens of $\mathrm{MHz}$ ), which enhances the correlation between nonlinear phase shifts of neighbouring subcarriers. With this technique, the fiber nonlinearity impairments due to the Kerr effect in OFDM systems can be effectively compensated without the complexity of DBP or 50\% loss in capacity of PC-TW. The technique proposed here can be effectively implemented in both single polarization and PMD systems, in both single channel and WDM systems. In other words, nonlinearity compensation using PCPs offers a simple, easy implementation applicable to any optical links where the level of nonlinear compensation may be readily tuned by selecting an appropriate number of PCPs.

\section{PHASE CONJUGATED PILOTS FOR FIBER NONLINEARITY} COMPENSATION

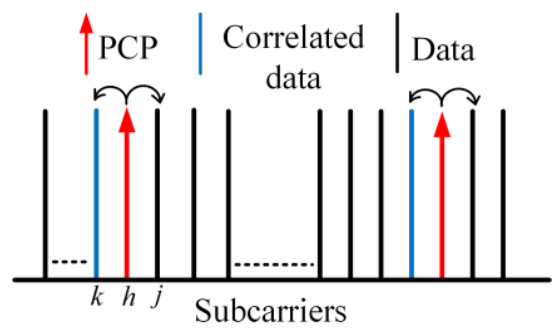

Fig. 1: Inserting phase-conjugated pilots for fibre nonlinearity compensation

Since the frequency spacing in an OFDM system is often small $[18,19]$ (tens of $\mathrm{MHz}$ ) compared to the phase matching bandwidth of the link (few GHz [20]), at the end of the optical link, the nonlinear phase shifts on adjacent subcarriers will be strongly correlated. This implies that nonlinear distortion experienced in one spectral region may be used to estimate the distortion in other closely space regions, as observed in pilot tone compensation schemes [21]. Thus nonlinear compensation can be achieved by sparsely inserting PCPs across the OFDM band.

The concept of inserting PCP is illustrated in the Fig. 1. Suppose the information symbol carried by the $\mathrm{k}^{\text {th }}$ subcarrier is $S_{k}=A_{k} \cdot \exp \left(j \cdot \phi_{k}\right)$ where $A_{k}$ and $\phi_{k}$ are the amplitude and the phase of this information symbol, then the phase conjugated symbol can be transmitted in the $\mathrm{h}^{\text {th }}$ subcarrier, $\mathrm{S}_{\mathrm{h}}=\mathrm{S}_{\mathrm{k}}{ }^{*}=\mathrm{A}_{\mathrm{k}} \cdot \exp \left(-\mathrm{j} \cdot \phi_{\mathrm{k}}\right)$, where $(.)^{*}$ represents complex conjugation. To simplify the exposition, we assume that during propagation nonlinear phase shifts, represented by $\theta_{\mathrm{k}}$ and $\theta_{\mathrm{h}}$, are added to these subcarriers. The received information symbols on the $\mathrm{k}^{\text {th }}$ and $\mathrm{h}^{\text {th }}$ subcarriers are $\mathrm{R}_{\mathrm{k}}=\mathrm{A}_{\mathrm{r}, \mathrm{k}} \cdot \exp \left(\mathrm{j} \cdot \phi_{\mathrm{k}}+\mathrm{j} \cdot \theta_{\mathrm{k}}\right) \quad$ and $\quad \mathrm{R}_{\mathrm{h}}=\mathrm{A}_{\mathrm{r}, \mathrm{h}} \cdot \exp \left(-\mathrm{j} \cdot \phi_{\mathrm{k}}+\mathrm{j} \cdot \theta_{\mathrm{h}}\right)$, respectively. If the frequency spacing between $k^{\text {th }}$ and $h^{\text {th }}$ subcarriers is small enough, the nonlinear phase shifts will be highly correlated, $\theta_{\mathrm{k}} \approx \theta_{\mathrm{h}}$ providing the opportunity of cancelling the nonlinear phase shift on the $\mathrm{k}^{\text {th }}$ subcarrier by averaging the received information symbol of a subcarrier and the subcarrier which carries its phase conjugate (after a second conjugation):

$$
\bar{R}_{k}=\left(R_{k}+R_{h}^{*}\right) / 2 \approx A_{r, k} \cdot \cos \left(\theta_{k}\right) \cdot \exp \left(j \phi_{k}\right)
$$

This compensation technique is often referred in the literature as coherent superposition $[11,22]$. Note that the nonlinear phase shift on the original subcarrier $k$ can be estimated as [23, 24]:

$$
\theta_{k}=\arg \left(R_{k} \cdot R_{h}\right) / 2
$$

Ideally, a data carrying subcarrier and its PCP should be closely spaced in frequency (adjacent) to maximize the level of correlation of the nonlinear phase shifts between these subcarriers. For those data carrying subcarriers which do not have PCPs, the nonlinear phase shift of the $j^{\text {th }}$ subcarrier can be estimated and compensated as:

$$
\begin{aligned}
& \theta_{j}=\arg \left(\sum_{k, h} \eta_{j k h} \cdot R_{k} \cdot R_{h}\right) / 2 \\
& \overline{R_{j}}=R_{j} \cdot e^{-j \cdot \theta_{j}}
\end{aligned}
$$

where $\eta_{j k h}$ is the FWM efficiency coefficient. In this paper $\eta_{j k h}$ is approximated either as 1 if $j$ is the closest subcarrier to $k$ or $h$ and 0 otherwise.

By applying this fiber compensation technique, the fiber nonlinearity phase shifts on data subcarriers in an OFDM system can be compensated without conjugating all pairs of subcarriers. In this system configuration, several data carrying subcarriers are placed between conjugate pairs. The nonlinear phase shifts for all of these subcarriers are similar as long as the frequency spacing is small. These nonlinear distortions can be compensated using the estimated nonlinear distortion on the closest pair of subcarrier data and phase conjugated pilot. As a result, one phase conjugated pilot can be used to compensate the nonlinear distortions on several subcarriers and the overhead due to phase conjugated pilots is relaxed and can be designed according to the requirement of a specific application.

Whilst the most accurate nonlinear compensation will be achieved by weighting and summing the nonlinear distortion estimated from all of the phase conjugate pairs, the nonlinear distortion on subcarriers which are not part of phase conjugate pairs can be estimated in various ways. The first approximation is to simply use the estimated nonlinear distortion from the nearest phase conjugate pair. The second 
approximation is to use a linear interpolation of the estimated nonlinear distortions from the two closest phase conjugate pairs (two points).

In common with PC-TW, the performance of a system based on PCP can be further improved with 50\% electrical dispersion pre-compensation (pre-EDC), which is applied to create a dispersion-symmetry along the transmission link. This dispersion map enhances the similarity between nonlinear distortions on subcarrier data and its phase conjugate, thus further improving the effectiveness of the proposed nonlinearity compensation scheme.

\section{Simulation}

As a proof of concept, we conducted simulation of the proposed PCP scheme for a single channel $112 \mathrm{~Gb} / \mathrm{s}$ PDM QPSK CO-OFDM transmission. The simulation setup is shown in the Fig. 2. The data stream was first divided into Xand y-polarizations, each of which was then mapped onto 1400 subcarriers using QPSK modulation format and subsequently transferred to the time domain by an IFFT of size 2048 while zeros occupying the remainder. Our standard simulation was as follows, although some parameters were varied to illustrate the salient features of this nonlinearity compensation scheme. The OFDM useful duration was $50 \mathrm{~ns}(20 \mathrm{MHz}$ subcarrier spacing), no cyclic prefix was added and we ignored here the effect of polarization mode dispersion. The fiber link comprised 80-km spans of standard single mode fiber (SSMF) with a loss parameter of $0.2 \mathrm{~dB} / \mathrm{km}$, nonlinearity coefficient of $1.22 \mathrm{~W}^{-1} \mathrm{~km}^{-1}$ and dispersion of $16 \mathrm{ps} / \mathrm{nm} / \mathrm{km}$. The span loss was compensated by Erbium-doped fiber amplifiers (EDFA) with $16 \mathrm{~dB}$ of gain and $6 \mathrm{~dB}$ noise figure. The amplified spontaneous emission (ASE) noise is added inline to ensure that the interaction between signal and noise [25] is correctly captured. The transmitter and receiver lasers had the same linewidth of $100 \mathrm{kHz}$. The simulated time window contained 100 OFDM symbols (560,000 bits).

The received signal after coherent reception was first resampled and converted from serial to parallel for further processing, including chromatic dispersion compensation using a frequency domain equalizer (OFDE) employing the overlap-and-save method, channel estimation and equalization with the assistance of initial training sequence (2 training symbols every 100 symbols) using zero forcing estimation with MIMO processing [26], and nonlinear phase noise (NLPN) estimation and compensation. In order to compensate for NLPN using PCPs, it is necessary to compensate for the common phase error (CPE) introduced by the lasers' phase noise and common phase shift due to the fiber nonlinearity first. To reduce the net overhead, this compensation is also achieved using all the PCPs as shown in [24], using a twostage compensation scheme. After CPE compensation, the nonlinear distortion of subcarriers data accompanied by PC pilots was compensated using Eq. 1 whilst the nonlinear distortions of other subcarriers were compensated using Eq. 3. In order to demonstrate effectiveness of the nonlinear noise cancellation scheme based on the coherent superposition of the
PCP pairs, we first turn off the ASE noise. The simulation results are shown in the Fig. 3 for a $1200 \mathrm{~km}$ optical link with $5 \mathrm{dBm}$ of the launch power. After coherent superposition, a dramatic reduction $(\sim 7 \mathrm{~dB})$ of the nonlinear signal distortion variance $\left(\sigma^{2}\right)$ was observed, indicating that the nonlinear distortion on a data carrying subcarrier and those of its PC are highly anticorrelated, especially if the frequency spacing is small.

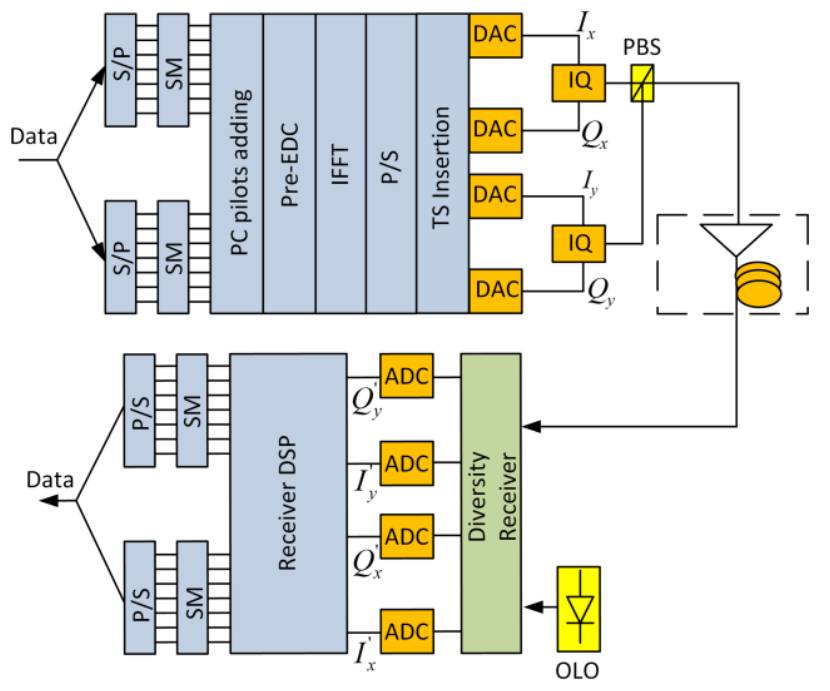

Fig. 2: Block diagram of $112 \mathrm{~Gb} / \mathrm{s}$ PDM CO-OFDM transmissions. S/P: serial/parallel conversion, P/S: parallel/serial conversion, SM: symbol mappings, TS: training symbol, DAC: digital-to-analog converter, ADC: analog-to-digital converter, I/Q: I/Q modulator, PBS: polarization beam spliter, OLO: optical local oscilator
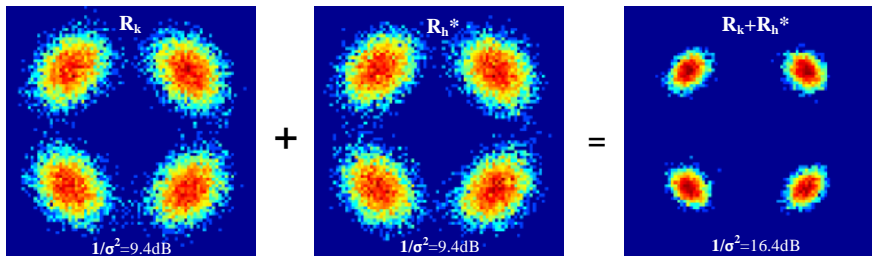

Fig. 3. Nonlinear noise cancellation based on the coherent superposition of PCP pairs. The transmission distance is $1200 \mathrm{~km}$, launch power is $5 \mathrm{dBm}$, ASE noise is not considered and 50\% pre-EDC is adopted.

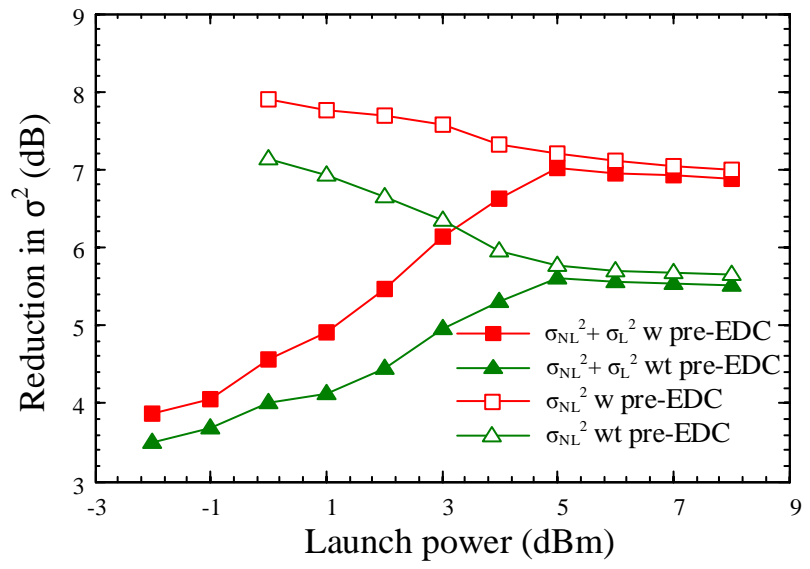

Fig. 4. Measured reduction of signal variance from both nonlinear distortion $\left(\sigma_{N L}{ }^{2}\right)$ and linear noise $\left(\sigma_{\mathrm{L}}^{2}\right)$ as a function of the launch power, in systems with and without $50 \%$ pre-EDC. The transmission distance is $1200 \mathrm{~km}$, ASE noise is included. 
Figure 4 plots the reduction in the signal variance $\left(\sigma^{2}\right)$, which is equivalent to the signal-to-noise ratio (SNR) improvement, when coherent superposition is applied for PCP pairs in systems with and without 50\% pre-EDC (red and green respectively). The difference between the open and solid symbols illustrates the impact of ASE noise. When the launch power is small, the dominant limiting factor in the system is the ASE noise. As a result, the coherent superposition of the PCP pairs using 50\% pilots offers around $\sim 3 \mathrm{~dB}$ reduction of the signal variance, as expected from the linear effects of coherent superposition of two copies of the same signal. However, with increasing launch powers a larger reduction in $\sigma^{2}$ eventually occurs, indicating the maximum effectiveness of the proposed nonlinear noise cancellation scheme. Note that in this regime, there is little impact from the addition of ASE noise suggesting that the system is limited by compensation accuracy rather than the fundamental parametric noise amplification process [25]. When 50\% pre-EDC is performed, the reduction of $\sigma^{2}$ is further enhanced, reaching around $7 \mathrm{~dB}$ at a high level of the launch power. When considering only the nonlinear noise distortion, an even higher reduction of $\sim 8 \mathrm{~dB}$ can be observed.

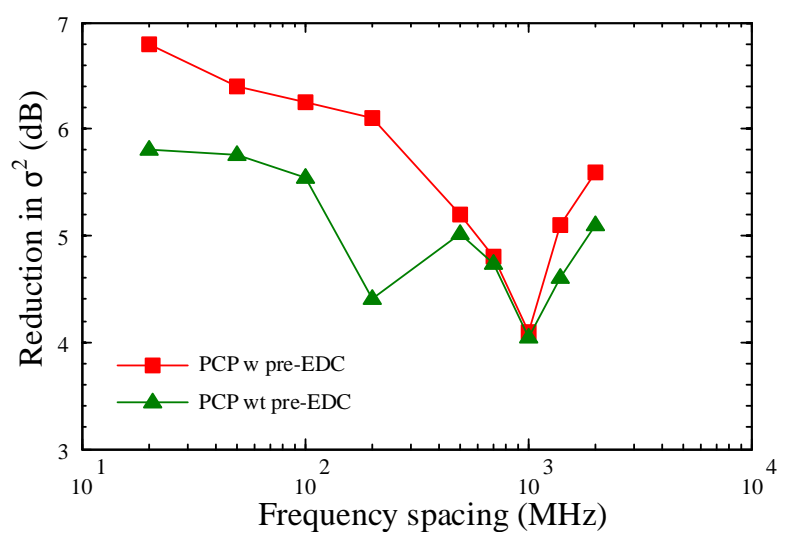

Fig. 5. Measured reduction of signal variance as a function of the frequency spacing. The transmission distance is $1200 \mathrm{~km}$, the launch power is $5 \mathrm{~dB}$, ASE noise is included and the number of subcarriers is varied, keeping the same data rate $(112 \mathrm{~Gb} / \mathrm{s})$.

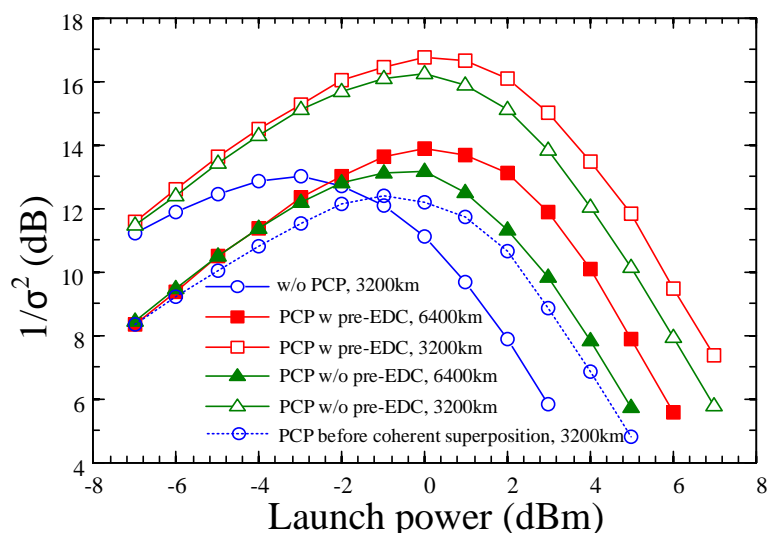

Fig. 6. Nonlinear cancellation using coherent superposition of PCP pairs in long-haul $56 \mathrm{~Gb} / \mathrm{s}$ (net data rate) CO-OFDM transmission. The transmission distances are $3200 \mathrm{~km}$ (open symbols) and $6400 \mathrm{~km}$ (closed symbols).

As mentioned before, the proposed PCP compensation scheme relies on the correlation of nonlinear phase shifts of the data carrying subcarriers and PCPs; the effectiveness of this scheme depends strongly on the subcarrier frequency spacing. Figure 5 shows the reduction of signal variance $\sigma^{2}$ as a function of the subcarrier frequency spacing (by varying the number of the subcarriers). As expected, when the subcarrier frequency spacing increases, the benefit of coherent superposition scheme tends to decrease, from around $7 \mathrm{~dB}$ at a frequency spacing of $20 \mathrm{MHz}$ to around $4 \mathrm{~dB}$ at a frequency spacing of $1 \mathrm{GHz}$ (for the case of $50 \%$ pre-EDC). The $3 \mathrm{~dB}$ nonlinear interaction bandwidth of the simulated system was around 1.1 GHz. However, we attribute the rapid dip in performance around $1 \mathrm{GHz}$ (and $200 \mathrm{MHz}$ without pre-dispersion) to a quasi-phase matching process $[20,25,27]$ rather than a specific resonance with this bandwidth.
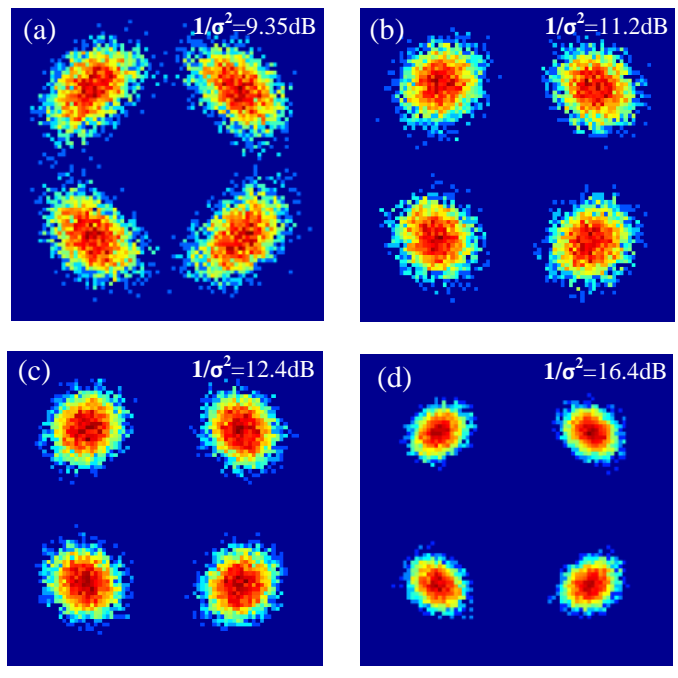

Fig. 7. Received constellation diagrams in $112 \mathrm{~Gb} / \mathrm{s}$ PDM CO-OFDM systems without (with the same bandwidth, before CS) (a) and with PCPs for fiber nonlinearity compensation (b-12.5\%, c - 25\%, d - 50\% overhead). The Transmission distance is $1200 \mathrm{~km}$, the launch power is $5 \mathrm{dBm}$.

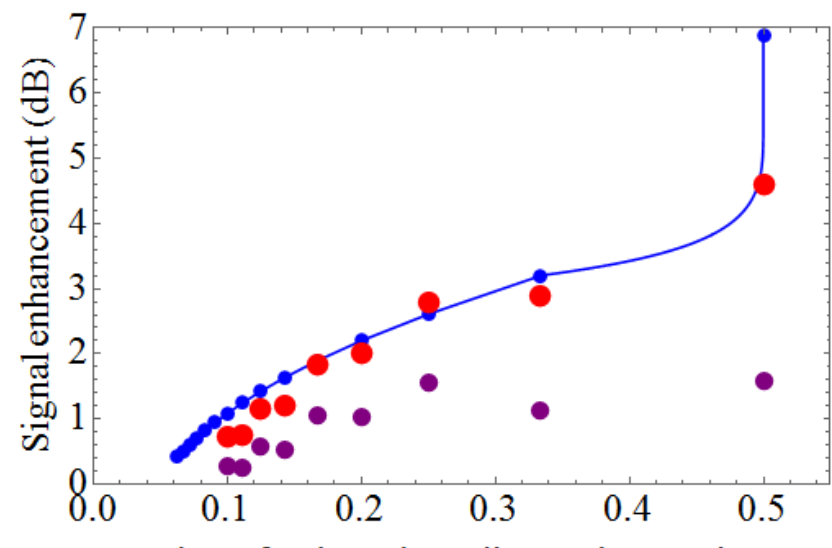

Fraction of subcarriers allocated as conjugates

Fig. 8. Signal enhancement of a $3,200 \mathrm{~km}$ PDM NGI CO-OFDM system at the optimum launch power as a function of the fraction of subcarriers allocated as phase conjugate pilots, showing measured reduction in signal variance (red symbols), net gain after subtraction of overhead (purple) and predicted signal to noise ratio gain (blue symbols).

The effective SNR of $112 \mathrm{~Gb} / \mathrm{s}$ OFDM systems with $50 \%$ PCPs and without any PCPs are compared in Fig. 6. Note that, 


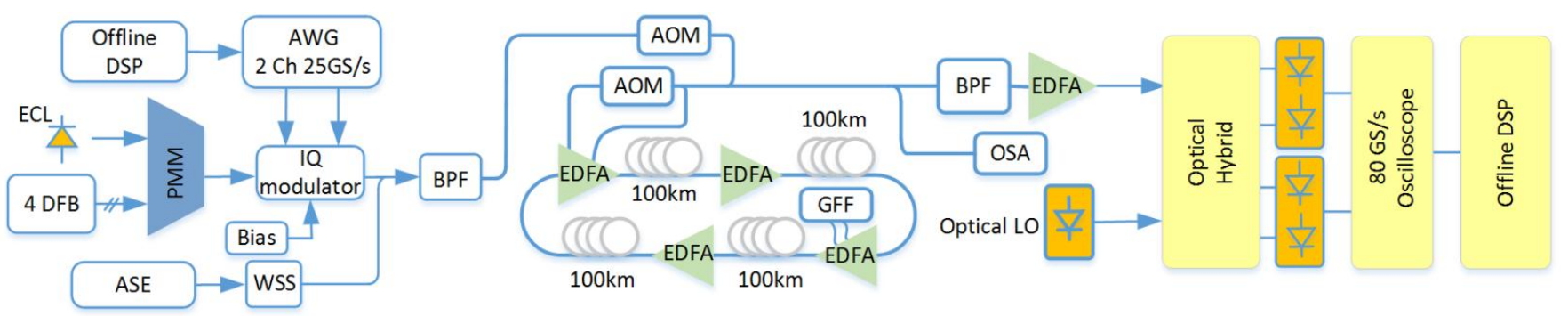

Fig. 9: Schematic of experimental setup of WDM CO-OFDM transmission with PCPs for fibre nonlinearity compensation. ECL: external cavity laser, PMM: polarization maintaining multiplexer, WSS: Wavelength Selective Switch, DFB: distributed feedback laser, BPF: band-pass filter (optical), AOM: acousto-optic modulator, GFF: gain flatten filter, OSA: optical spectrum analyser, LO: local oscillator.

the spectral efficiency is reduced by a factor of 2 when $50 \%$ of the subcarriers are transmitted as PCPs. In Fig. 6, by combining pre-EDC and PCP techniques, a reduction of around $4.5 \mathrm{~dB}$ in the signal variance can be achieved at a transmission distance of $3200 \mathrm{~km}$ at the cost of $50 \%$ overhead. The nonlinear threshold is also increased by $9 \mathrm{~dB}$ with PCP compensation. This result clearly indicates that a substantial fraction of the nonlinear distortion can be mitigated by coherently adding the phase conjugated pilot and its correlated data subcarrier. As a result of this improvement, a longer transmission distance can be achieved. In Fig. 6 the effective SNR of system with $50 \%$ PCP after $6400 \mathrm{~km}$ of transmission distance is also plotted for comparison purpose. As can be seen, this system still offers around $1.5 \mathrm{~dB}$ advantage in performance in comparison with OFDM system without PCP after $3200 \mathrm{~km}$ of transmission distance. This comparison indicates that the product of spectral efficiency and transmission distance can be significantly increased with PCP techniques, and is consistent with results obtained for PC-TW. This implementation offers excellent performance but it requires 50\% overhead. The required overhead can be reduced by using the estimated nonlinear distortion on one pair of subcarrier data and its PCP to compensate the nonlinear distortions on other subcarriers. Specifically, one PCP can be used to compensate the nonlinear distortion of 2, 3, 4 or more data subcarriers at the cost of $33 \%, 25 \%, 20 \%$ or smaller overhead respectively. In Fig. 7 the received constellation diagrams of systems with and without PCPs for fiber nonlinearity compensation are shown for different values of PCP overhead with the launch power deliberately set in the highly nonlinear region $(+5 \mathrm{dBm})$. The trade-off between overhead due to PCPs and performance can be clearly observed. A better performance comes with the cost of larger overhead due to the transmission of additional PCPs.

The reduction in the signal variance (in $\mathrm{dB}$ ) at the optimum launch power (difference of the minimum achievable $\sigma^{2}$ in systems without and with PCPs) and the net benefit in $\mathrm{dB}$ (after extracting the spectral efficiency reduction due to the PCPs) as a function of the overhead due to PCPs are shown in the Fig. 8. With $50 \%, 33 \%$, and $20 \%$ overhead the achievable reduction in $\sigma^{2}$ are $4.6 \mathrm{~dB}, 3.2 \mathrm{~dB}$ and $2.1 \mathrm{~dB}$ respectively, or approximately $0.1 \mathrm{~dB}$ per $1 \%$ of overhead. We believe that the reduction in $\sigma^{2}$ enhancement as the overhead is reduced is initially due to lower coherent gain (a smaller number of subcarriers have the $>3 \mathrm{~dB}$ benefit of coherent superposition), but eventually the subcarriers become spaced by more than the FWM efficiency bandwidth after which the nonlinear compensation starts to reduce. This is confirmed by a theoretical estimation (shown in blue). To obtain this estimation the normalized difference in nonlinear distortion from FWM [20] was computed for all possible subcarrier triplets who's nonlinear distortions fall on either the carrier or it's conjugate. The sum of these nonlinearity compensation errors was used to estimate the level to which inter subcarrier nonlinearity is suppressed in the calculation of nonlinear noise. To give an upper bound on the compensation performance we assume that parametric noise amplification [25] is not compensated by the conjugates. The optimum signal to noise ratio was used to calculate the BER for subcarriers with and without a phase conjugate assuming that coherent superposition enabled a $3 \mathrm{~dB}$ enhancement in signal to noise ratio [11]. Finally the mean BER was used to calculate the required SNR to give the same performance without conjugate pilots. The curve fit between 33\% and 50\% conjugates assumes a linear interpolation of the nonlinear compensation error, but is dominated by the increasing effectiveness of the coherent superposition. An excellent fit is observed over a wide range of configurations with the exception of the case where $50 \%$ of the subcarriers are phase conjugate pilots. We believe that this error is due to a reduced benefit of coherent superposition when the noise fields are no longer statistically independent, due to their parametric amplification by the signal. In a practical system, a minimum overhead for CPE (4-10\%) would be required, and this overhead may be used to provide a certain level of nonlinear compensation without additional overhead.

\section{EXPERIMENTAL DEMONSTRATION}

The experimental set-up is shown in Fig. 9. It comprised a laser grid of five standard DFBs on $100 \mathrm{GHz}$ grid which were substituted in turn by a $100 \mathrm{kHz}$ linewidth laser. The DFBs are located between 193.5 to $193.9 \mathrm{THz}$. Additional loading channels (10 GHz of bandwidth) were generated using an ASE source which were spectrally shaped using a wavelength selective switch (WSS) [28]. The twenty loading channels were spread symmetrically around the test wavelengths so that the total bandwidth of the transmitted signal was $2.5 \mathrm{THz}$. A wideband filter was used to filter out of band ASE noise at the transmitter. The transmission path was an acousto-optic modulator (AOM) based re-circulating loop consisting of 4 x $100 \mathrm{~km}$ spans of Sterlite OH-LITE (E) fibre, having 18.9 to $19.5 \mathrm{~dB}$ insertion loss. The loop switch was located in the 
mid-stage of the first EDFA and a gain flattening filter (GFF) was placed in the mid stage of the third EDFA. After propagation the signal was filtered using a $4.2 \mathrm{~nm}$ flat topped filter and coherently detected. The received electrical signals were then sampled by a real-time oscilloscope at $80 \mathrm{GS} / \mathrm{s}$ and processed offline in MATLAB.

The OFDM signal (400 symbols each of 20.48 ns length, $2 \%$ cyclic prefix) encoded with QPSK modulation format was generated offline in MATLAB using an IFFT size of 512, where 210 subcarriers were filled with data and the remainder zeros giving a line rate of $20 \mathrm{~Gb} / \mathrm{s}(18.2 \mathrm{~Gb} / \mathrm{s}$ after cyclic prefix and FEC overhead are removed). When $25 \%, 33.3 \%$ and $50 \%$ of OFDM subcarriers are transmitted with its PCPs the net data rates were $13.65 \mathrm{~Gb} / \mathrm{s}, 12.12 \mathrm{~Gb} / \mathrm{s}$ and $9.1 \mathrm{~Gb} / \mathrm{s}$ respectively. In this work, in order to maximize the similarity between nonlinear phase noises on data subcarrier and PCP, data subcarrier and its PCP were placed next to each other. The DSP at the receiver included chromatic dispersion compensation using an overlapped frequency domain equalizer with overlap-and-save method, channel estimation and equalization with the assistance of initial training sequence (2 training symbols every 100 symbols), CPE compensation with the help of the PCPs [23] or 16 pilot subcarriers if PCPs were not transmitted, fibre nonlinearity compensation as described above, and symbol detection. The system performance was evaluated directly from the BER by processing 10 recorded traces $\left(\sim 10^{6}\right.$ bits). The measured BER is then converted to an equivalent "Gaussian noise" Q-factor in $\mathrm{dB}$ using the expression $[29,30]$ :

$$
Q_{B E R}=20 \log \left[\sqrt{2} \cdot \operatorname{efrc}^{-1}(2 B E R)\right]
$$

Where $\operatorname{erfc}^{-1}$ is the inverse complementary error function.
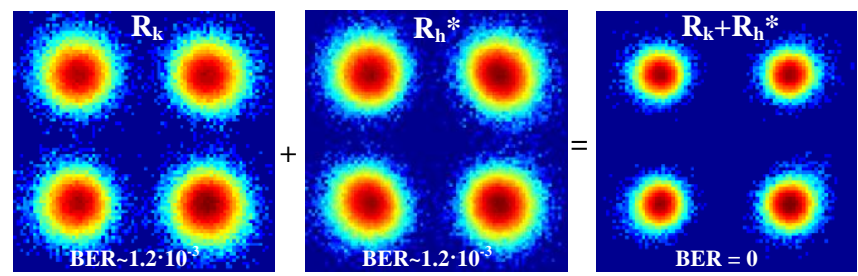

Fig. 10. Cancelllation of the nonlinear distortions by CS of subcarriers with its couterpart PCPs in WDM CO-OFDM transmission, $800 \mathrm{~km}$ of distance, the launch power (per/ch) was $0 \mathrm{dBm}$.

The effectiveness of the proposed PCP compensation scheme for fiber nonlinearity compensation in CO-OFDM is shown in Fig. 10, for the center channel. Before coherent superposition, the measured BER was $\sim 1.2 \cdot 10^{-3}$. However, after coherent superposition error free transmission was observed. This result clearly indicates that a substantial fraction of the nonlinear distortion, including both intra and inter-channel distortions, can be effectively compensated using coherent superposition of the data carrying subcarriers and the PCPs.

It can be seen in the Fig. 11 (a), that by transmitting $50 \%$ of OFDM subcarriers as PCPs a dramatic ( 4 dB) improvement in the system's Q-factor was achieved (after $3200 \mathrm{~km}$ of the transmission distance), which agrees well with the simulation result and is also comparable with results achieved with the conventional PC-TW technique [11]. The optimum launch power was increased by around $1 \mathrm{~dB}$.

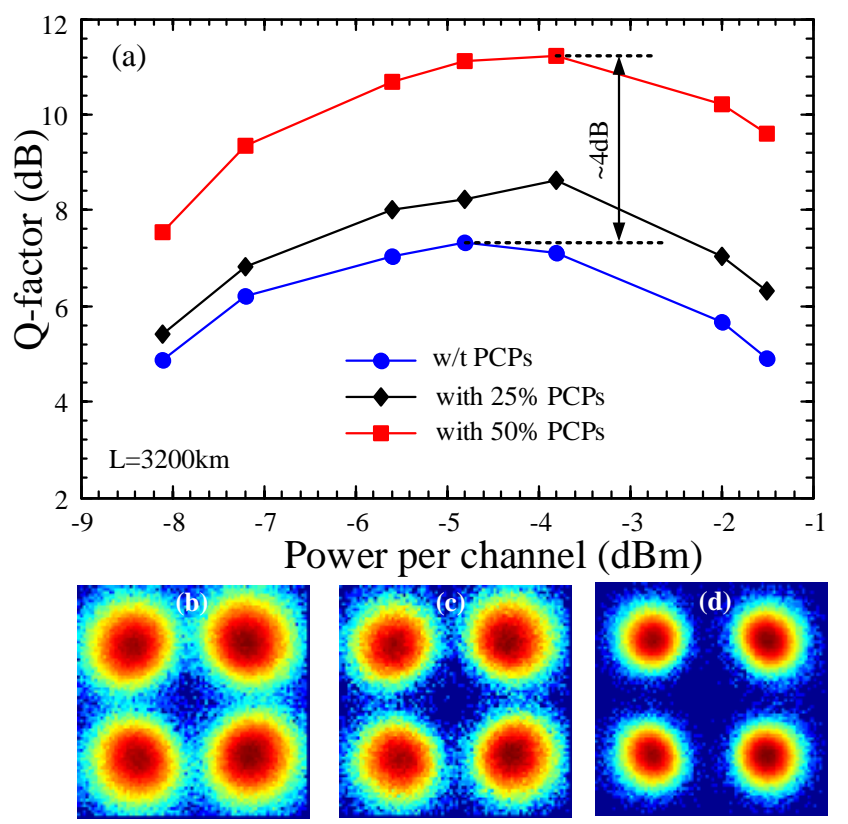

Fig. 11. Q-factor of the center channel as a function of the launch power in system with and without PCPs for fibre nonlinearity compensation (a) and constellation diagrams at Pin $=-1.5 \mathrm{dBm}$ for the cases of without PCPs (b) and with $25 \%, 50 \%$ of PCPs (c and d) respectively.

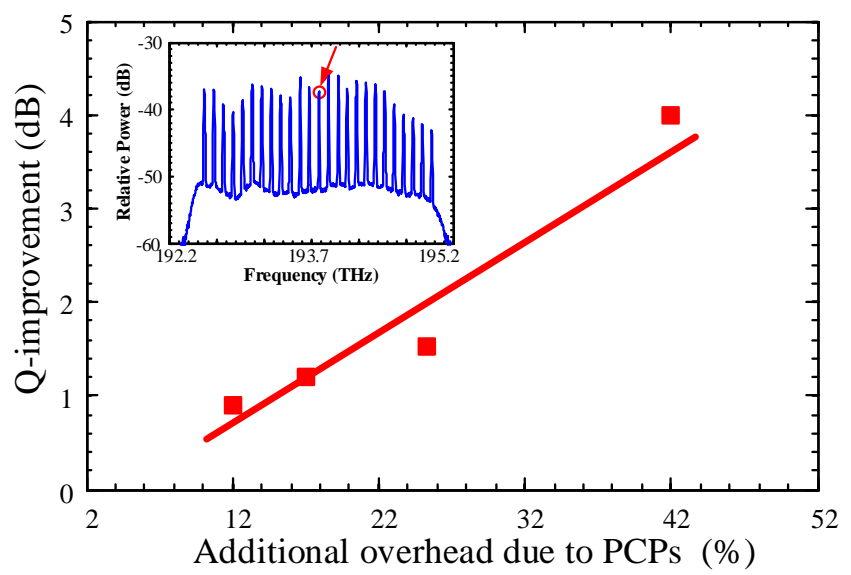

Fig. 12. Performance gain as a function of the additional overhead due to PCPs for the center channel, after $3200 \mathrm{~km}$ of distance. Without PCPs, an overhead of $\sim 8 \%$ was required for CPE compensation.

As mentioned before, the overhead can be reduced by using a smaller number of PCP. Specifically, one PCP can be used for $2,3,4$ or more data subcarriers at the cost of $33 \%, 25 \%$, $20 \%$ or smaller overhead respectively. When the PCP overhead was reduced to $25 \%$, a performance improvement of around $1.5 \mathrm{~dB}$ was still achieved (Fig. 11(a)). This result confirms the possibility of using one PCP to compensate the nonlinear distortions of several data carrying subcarriers. Figure 12 plots the Q-factor improvement as a function of the additional overhead due to the PCP, showing the trade-off between PCP overhead and performance gain. Since when PCPs were not transmitted, $8 \%$ of OFDM subcarriers were allocated for phase noise estimation, the additional overhead for nonlinear compensations were $12 \%, 17 \%, 25 \%$ and $42 \%$ and the performance gains were $0.9,1.2,1.5$ and $4 \mathrm{~dB}$ respectively. This result clearly shows the flexibility of the proposed PCP fibre nonlinearity compensation technique, 
allowing the number of PCP to be chosen to meet the performance requirement.

\section{CONCLUSION}

We have demonstrated a novel fiber nonlinearity compensation technique for CO-OFDM systems based on the transmission of phase-conjugated pilots, which can be effectively applied in both single polarization and PMD systems, in both single channel and WDM systems. The effectiveness of this technique was demonstrated by numerical simulation and laboratory experiment, showing that a direct improvement of up to $4 \mathrm{~dB}$ can be achieved. In addition, with this scheme, one phase conjugated pilot can be used to compensate the nonlinear distortions on several subcarriers and thus, the overhead due to phase conjugated pilots can be chosen according to the requirement of a specific application, offering the highest flexibility in implementations.

\section{ACKNOWLEDGMENT}

This work was partially supported by the UK EPSRC programme Grants UNLOC and PEACE (EP/J017582/1 and EP/L000091/1) and the European Commission's 7th Framework Programme FP/2007-2013 grants 258033 (MODE-GAP) and 318415 (FOX-C), and the support of Sterlite Technologies and Finisar is gratefully acknowledged.

\section{REFERENCES}

[1] C. E. Shannon, "A Mathematical Theory of Communication," The Bell System Technical Journal, vol. 27, pp. 379-432, 1948.

[2] R. Essiambre, G. Kramer, P. J. Winzer, G. J. Foschini, and B. Goebel, "Capacity Limits of Optical Fiber Networks," Lightwave Technology, Journal of, vol. 28, pp. 662-701, 2010.

[3] P. P. Mitra, Stark, Jason B., "Nonlinear limits to the information capacity of optical fibre communications," Nature, vol. 411, pp. 10271030, 2001.

[4] S. T. Le, J. E. Prilepsky, and S. K. Turitsyn, "Nonlinear inverse synthesis for high spectral efficiency transmission in optical fibers," Opt. Express, pp. 26720-26741, 2014.

[5] E. Ip and J. M. Kahn, "Compensation of Dispersion and Nonlinear Impairments Using Digital Backpropagation," Journal of Lightwave Technology, vol. 26, pp. 3416-3425, 2008/10/15 2008.

[6] C. Xi, L. Xiang, S. Chandrasekhar, B. Zhu, and R. W. Tkach, "Experimental demonstration of fiber nonlinearity mitigation using digital phase conjugation," presented in OFC 2012, pp. 1-3.

[7] S. L. Jansen, D. Van den Borne, B. Spinnler, S. Calabro, H. Suche, P. M. Krummrich, et al., "Optical phase conjugation for ultra long-haul phase-shift-keyed transmission," Lightwave Technology, Journal of, vol. 24, pp. 54-64, 2006.

[8] D. M. Pepper and A. Yariv, "Compensation for phase distortions in nonlinear media by phase conjugation," Optics Letters, vol. 5, pp. 5960, 1980/02/01 1980 .

[9] I. Phillips, M. Tan, M. F. Stephens, M. McCarthy, E. Giacoumidis, S. Sygletos, et al., "Exceeding the Nonlinear-Shannon Limit using Raman Laser Based Amplification and Optical Phase Conjugation," OFC, San Francisco, California, 2014, p. M3C.1.

[10] S. Watanabe, S. Kaneko, and T. Chikama, "Long-Haul Fiber Transmission Using Optical Phase Conjugation," Optical Fiber Technology, vol. 2, pp. 169-178, 4// 1996.

[11] X. Liu, R. A. Chraplyvy, P. J. Winzer, W. R. Tkach, and S. Chandrasekhar, "Phase-conjugated twin waves for communication beyond the Kerr nonlinearity limit," Nat Photon, vol. 7, pp. 560-568, 2013.

[12] T. Yoshida, T. Sugihara, K. Ishida, and T. Mizuochi, "Spectrallyefficient Dual Phase-Conjugate Twin Waves with Orthogonally
Multiplexed Quadrature Pulse-shaped Signals," in Optical Fiber Communication Conference, San Francisco, California, 2014, p. M3C.6.

[13] S. T. Le, E. Giacoumidis, N. Doran, A. D. Ellis, and S. K. Turitsyn, "Phase-conjugated Subcarrier Coding for Fibre Nonlinearity Mitigation in CO-OFDM Transmission," presented at the ECOC, Cannes, France, paper We.3.3.2, 2014.

[14] X. Liu, A. R. Chraplyvy, R. W. Tkach, and P. J. Winzer, "Communication Through Phase-Conjugated Optical Variants," USA Patent, 2012.

[15] Y. Tian, Y.-K. Huang, S. Zhang, P. R. Prucnal, and T. Wang, "Demonstration of digital phase-sensitive boosting to extend signal reach for long-haul WDM systems using optical phase-conjugated copy," Optics Express, vol. 21, pp. 5099-5106, 2013/02/25 2013.

[16] X. Yi, X. Chen, D. Sharma, C. Li, M. Luo, Q. Yang, et al., "Digital coherent superposition of optical OFDM subcarrier pairs with Hermitian symmetry for phase noise mitigation," Optics Express, vol. 22, pp. 13454-13459, 2014/06/02 2014.

[17] S. T. Le, M. E. McCarthy, N. M. Suibhne, A. D. Ellis, and S. K. Turitsyn, "Phase-conjugated Pilots for Fibre Nonlinearity Compensation in CO-OFDM Transmission," presented at the ECOC, Cannes, France, paper We.2.3.1, 2014.

[18] S. T. Le, K. Blow, and S. Turitsyn, "Power pre-emphasis for suppression of FWM in coherent optical OFDM transmission," Optics Express, vol. 22, pp. 7238-7248, 2014/03/24 2014.

[19] F. Buchali, R. Dischler, and X. Liu, "Optical OFDM: A promising high-speed optical transport technology," Bell Labs Technical Journal, vol. 14, pp. 125-146, 2009.

[20] D. A. Cleland, A. D. Ellis, and C. H. F. Sturrock, "Precise modelling of four wave mixing products over $400 \mathrm{~km}$ of step-index fibre," Electronics Letters, vol. 28, pp. 1171-1173, 1992.

[21] B. Inan, S. Randel, S. L. Jansen, A. Lobato, S. Adhikari, and N. Hanik, "Pilot-tone-based nonlinearity compensation for optical OFDM systems," presented in ECOC 2010, pp. 1-3.

[22] X. Liu, S. Chandrasekhar, P. J. Winzer, A. R. Chraplyvy, R. W. Tkach, B. Zhu, et al., "Scrambled coherent superposition for enhanced optical fiber communication in the nonlinear transmission regime," Optics Express, vol. 20, pp. 19088-19095, 2012/08/13 2012.

[23] S. T. Le, T. Kanesan, M. McCarthy, E. Giacoumidis, I. Phillips, M. F. Stephens, et al., "Experimental Demonstration of Data-dependent Pilot-aided Phase Noise Estimation for CO-OFDM," in Optical Fiber Communication Conference, San Francisco, California, 2014, p. Tu3G.4.

[24] L. Son Thai, T. Kanesan, E. Giacoumidis, N. J. Doran, and A. D. Ellis, "Quasi-Pilot Aided Phase Noise Estimation for Coherent Optical OFDM Systems," Photonics Technology Letters, IEEE, vol. 26, pp. 504-507, 2014.

[25] D. Rafique and A. D. Ellis, "Impact of signal-ASE four-wave mixing on the effectiveness of digital back-propagation in $112 \mathrm{~Gb} / \mathrm{s}$ PMQPSK systems," Optics Express, vol. 19, pp. 3449-3454, 2011/02/14 2011.

[26] S. L. Jansen, I. Morita, T. C. W. Schenk, and H. Tanaka, "121.9-Gb/s PDM-OFDM Transmission With 2-b/s/Hz Spectral Efficiency Over $1000 \mathrm{~km}$ of SSMF," Lightwave Technology, Journal of, vol. 27, pp. 177-188, 2009.

[27] A. D. Ellis and W. A. Stallard, "Four wave mixing in ultra long transmission systems incorporating linear amplifiers," in Non-Linear Effects in Fibre Communications, IEE Colloquium on, 1990, pp. 6/1$6 / 4$.

[28] M. E. McCarthy, N. M. Suibhne, S. T. Le, P. Harper, and A. D. Ellis, "High Spectral Efficiency Transmission Emulation for Non-Linear Transmission Performance Estimation for High Order Modulation Formats," presented at the ECOC, Cannes, France, paper P.5.7, 2014.

[29] S. T. Le, K. J. Blow, V. K. Menzentsev, and S. K. Turitsyn, "Comparison of numerical bit error rate estimation methods in 112Gbs QPSK CO-OFDM transmission," in Optical Communication (ECOC 2013), 39th European Conference and Exhibition on, 2013, pp. 1-3.

[30] S. T. Le, K. J. Blow, V. K. Mezentsev, and S. K. Turitsyn, "Bit Error Rate Estimation Methods for QPSK CO-OFDM Transmission," Lightwave Technology, Journal of, vol. 32, pp. 2951-2959, 2014. 